\title{
Capillary haemangioma presenting as a lung pseudocyst
}

\author{
J J Bowyer, M Sheppard
}

\begin{abstract}
A girl who developed a lung cyst at 24 hours of age during gentle ventilation for respiratory distress syndrome is reported. Instead of resolving as expected of a pseudocyst it continued to expand. Resection at 1 year of age showed a cyst entirely surrounded by capillary haemangioma. Aspiration of this cyst would have been dangerous.
\end{abstract}

Pulmonary pseudocyst formation is a recognised complication of ventilation in neonates with respiratory distress syndrome. We report an infant where the cyst did not resolve as expected. ${ }^{1}$

\section{Case report}

A girl of 30 weeks' gestation and birth weight $1200 \mathrm{~g}$ was born by emergency caesarean section for abruption. Dates were confirmed by an early scan and the pregnancy had been normal until slowing of fetal growth at 28 weeks. She was intubated and ventilated for five minutes initially and reintubated at 15 minutes for recession and cyanosis in $60 \%$ oxygen.

Blood gas concentrations were satisfactory and peak pressures no higher than $16 \mathrm{~cm}$ $\mathrm{H}_{2} \mathrm{O}$ were used for ventilation. Hypotension was treated with fresh frozen plasma. The first chest radiograph showed mild hyaline membrane disease, and the second at 18 hours showed the first suggestion of a bulla in the apex of the right lower lobe posteriorly in association with a right pneumothorax, which was drained (fig 1A). The air leak continued for five days, and she was extubated on the sixth day and the drain removed on the seventh. Serial radiographs showed pulmonary interstitial emphysema and a progressive increase in the size of the bulla (fig 1B). She remained somewhat tachypnoeic and required oxygen until the 57 th day. At three months of age she developed a capillary haemangioma on the scalp and one on the buccal mucosa but she has not had any telangectasia.
Department of Paediatrics, St Peter's Hospital, Guildford Road, Chertsey, Surrey KT16 0PZ J J Bowyer Department of Pathology, Brompton Hospital, London M Sheppard Correspondence to: Dr Bowyer. Accepted 10 May 1990
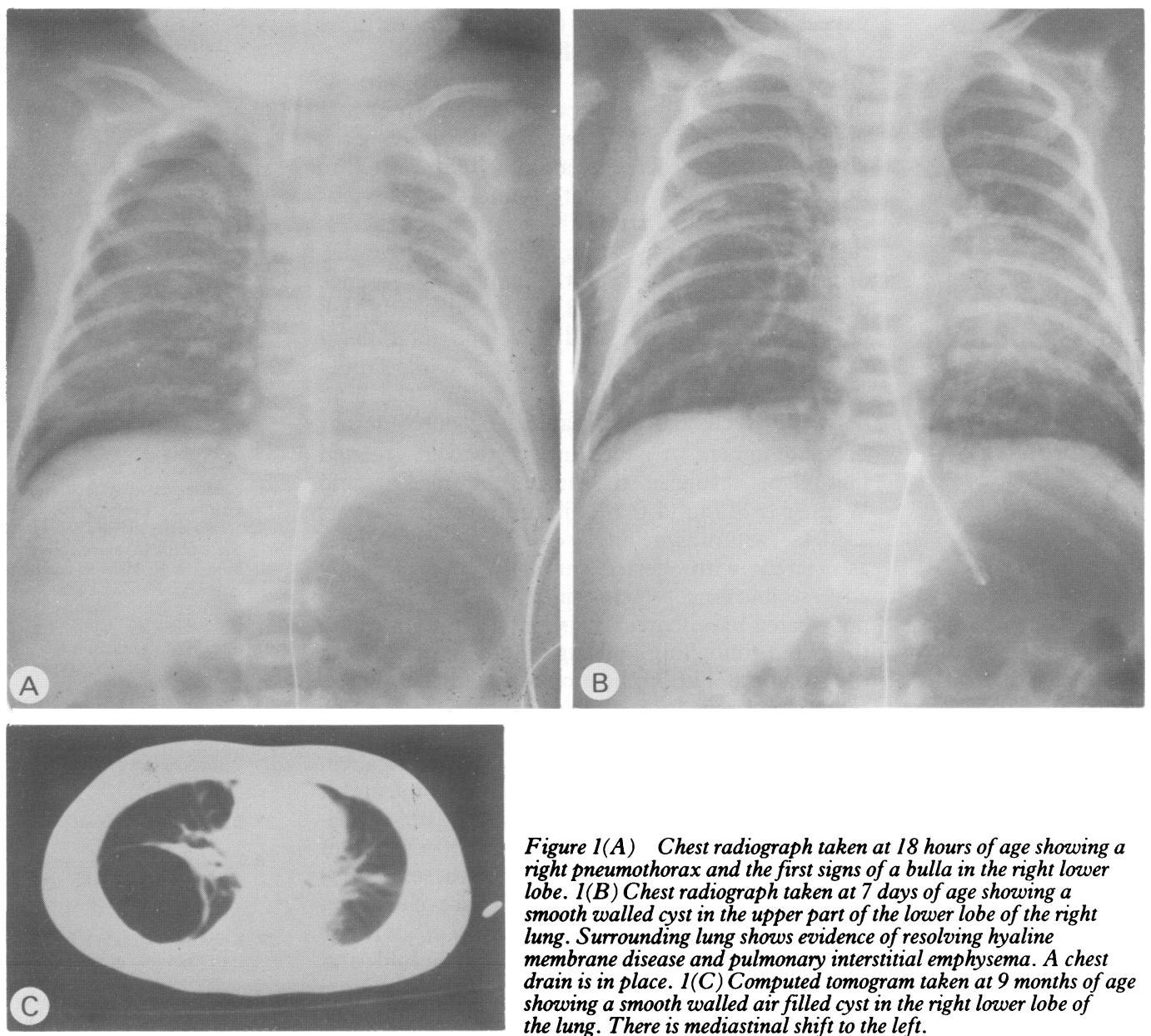

Figure 1(A) Chest radiograph taken at 18 hours of age showing a right pneumothorax and the first signs of a bulla in the right lower lobe. $1(B)$ Chest radiograph taken at 7 days of age showing a smooth walled cyst in the upper part of the lower lobe of the right lung. Surrounding lung shows evidence of resolving hyaline membrane disease and pulmonary interstitial emphysema. A chest drain is in place. $1(C)$ Computed tomogram taken at 9 months of age showing a smooth walled air filled cyst in the right lower lobe of the lung. There is mediastinal shift to the left. 

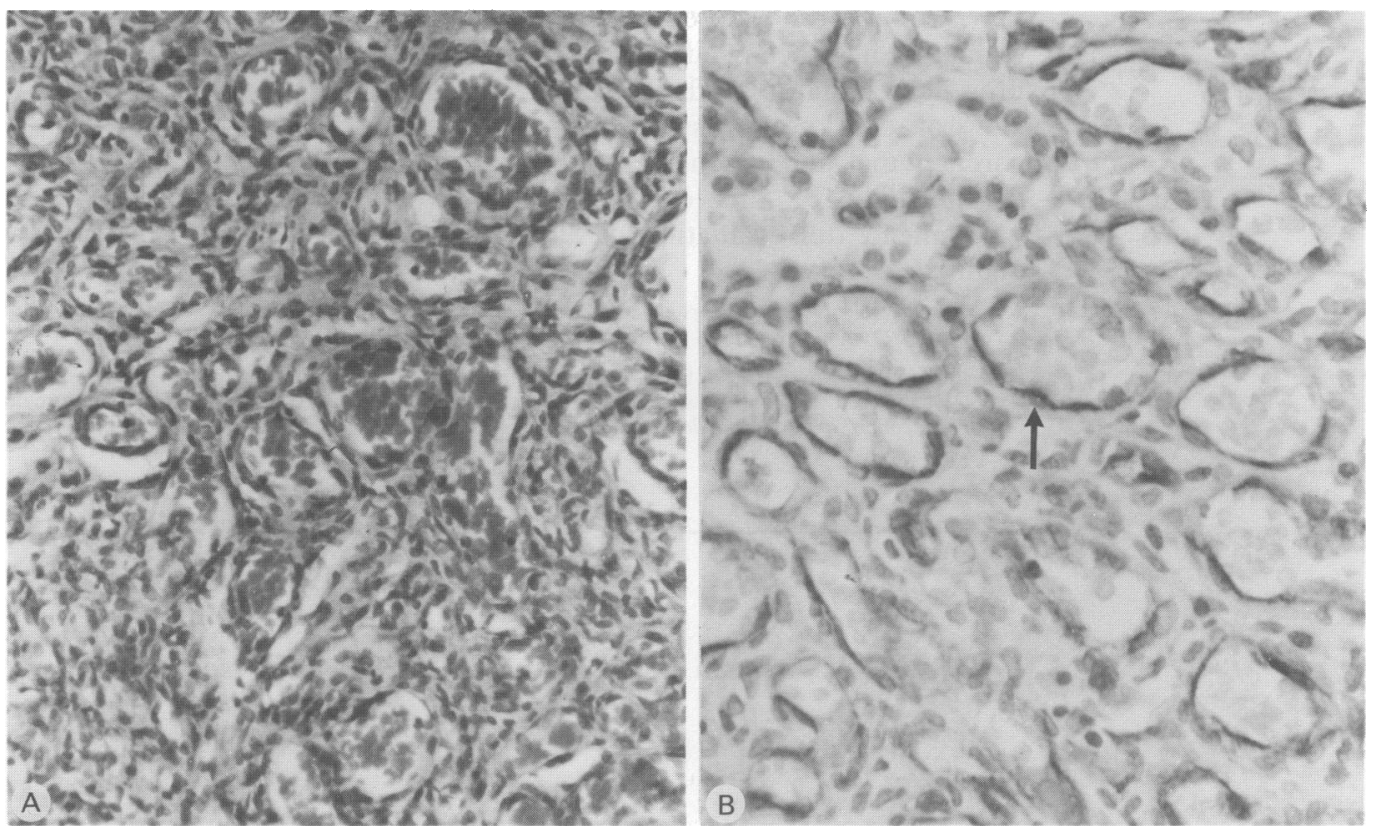

Figure 2(A) Wall of cyst containing multiple capillaries lined by endothelial cells (stained with haemotoxylin and eosin $\times 400)$. 2(B) Capillaries lined by endothelial cells immunoreactive for factor VIII (arrow) (avidin-biotin complex method $\times 400$ ).

At 9 months computed tomography showed a tense cyst in the right lower lobe which was compressing the right upper lobe and causing mediastinal shift (fig 1C). There was a second, smaller cyst just to the left of the hilum and consolidation of the left lower lobe. A ventilation-perfusion scan showed a matched deficit corresponding to the cyst but no abnormality of vascular supply.

At operation at 1 year a tense intrapulmonary cyst, $6 \mathrm{~cm}$ diameter, distended the right lower lobe and spread onto both hilar and lateral surfaces encircling the hilar structures. The vascular connections of the lung were normal. The right lower lobe was resected but the cyst on the left was not explored.

\section{HISTOPATHOLOGY}

The specimen received was a segment of the right lower lobe of the lung measuring $8.5 \times 5$ $\times 2.5 \mathrm{~cm}$ with a previously incised cyst measuring $3 \mathrm{~cm}$ in the collapsed state. The cyst wall was smooth with some fibrous thickening in areas and was completely surrounded by lung parenchyma. No vascular or bronchial connection to the cyst could be seen. Microscopic analysis showed that the cyst wall was composed largely of multiple capillaries lined by plump endothelium (fig 2A), which stained positively for factor VIII related antigen (fig 2B). Capillaries varied in size, some were collapsed, others filled with blood. The inner lining of the cyst contained fibrous tissue but no respiratory or gastrointestinal epithelium and no mitotic activity was seen. Giant cell reaction, which might be expected in interstitial emphysema, was not seen. The capillaries infiltrated the surrounding lung irregularly for a short distance but the parenchyma appeared normal with no diffuse involvement by the angioma. A diagnosis of capillary haemangioma was made.

\section{Discussion}

Pulmonary pseudocyst formation is a recognised complication of ventilation in neonates with respiratory distress syndrome. Such cases are associated with the development of interstitial emphysema, pneumothoraces, and later bronchopulmonary dysplasia. ${ }^{1}$ Thus our initial impression in this case was that the cyst was a ventilation induced pseudocyst that would be expected to resolve spontaneously.

The increasing size of the cyst and evidence of compression of the surrounding lung led to the decision to remove the affected lobe. The finding of a capillary haemangioma entirely surrounding the cyst was unexpected and has not previously been reported.

True congenital lung cysts are usually either bronchogenic cysts, which are lined with respiratory epithelium, enteric cysts lined with gastrointestinal epithelium, or cystic adenomatoid malformations. ${ }^{2}$ These are characterised by cysts and bronchioles lined by ciliated columnar epithelium and goblet cells, and may become malignant. ${ }^{3}$ Even apparently simple congenital lung cysts may be associated with abnormal vasculature, either systemic arterial supply or anomalous venous return, and require full evaluation before surgery is planned. ${ }^{4}$

Vascular malformations in the lung are generally cavernous haemangiomata with large channels of communication between pulmonary arteries and veins, commonly associated with hereditary haemorrhagic telangectasia. ${ }^{5}$ True capillary haemangiomata occurring in the bronchial submucosa may give rise to severe haemoptysis. ${ }^{6}$ One comparable case of vascular channels lying in the substance of the lung in association with an expanding cyst was reported in a 25 year old man, but there the cyst was lined with respiratory epithelium. ${ }^{7}$

Our finding of a capillary haemangioma totally surrounding a lung cyst appears to be 
new. Aspiration of pseudocysts in ventilated neonates has been advocated, ${ }^{8}$ but in the present case that might have been disastrous. In view of the known association of congenital parenchymal and vascular abnormalities, ${ }^{4}$ and in the light of this experience, we would recommend full evaluation of all cysts before planned surgery.

We are grateful to Dr $\mathrm{J}$ Warner and $\mathrm{Mr} \mathrm{P}$ Goldstraw for permission to report the patient in their care.

1 Williams DW, Merten DF, Effman EL, Scatliff JH. Ventilator induced pulmonary pseudocysts in preterm neonates. American fournal of Radiology 1988;150:885-7.
2 Spencer $\mathbf{H}$. Congenital abnormalities of the lung, pulmonary vessels and lymphatics. Pathology of the lung. 4th Ed. London: Pergamon Press, 1985:79-130.

3 Sheffield EA, Addis BJ, Corrin B, McCabe MM. Epithelial hyperplasia and malignant change in congenital lung cysts. f Clin Pathol 1987;40:612-4.

4 Clements BS, Warner JO. Congenital bronchopulmonary vascular malformations: clinical application of a simple anatomical approach in 25 cases. Thorax 1987;42:409-16.
Dunnill MS. Rare pulmonary tumours. Pulmonary pathology. London: Churchill Livingstone, 1987:353-6.

London: Churchill Livingstone, 1987:353-6.
Masson RG, Altose MD, Maycock RL. Isolated bronchial Masson RG, Altose MD, Maycock R
telangiectasia. Chest 1974;65:450-2.

7 Holden WE, Mulkey DD, Kessler S. Multiple peripheral lung cysts and haemoptysis in an otherwise asymptomatic adult. Am Rev Resp Dis 1982;126:930-2.

8 Roberton NRC. Treatment of cystic ventilator lung disease. Proceedings of the Royal Society of Medicine 1976;69:344-5.

\section{Forty years ago}

\section{Infantile cortical hyperostosis \\ (Caffey's syndrome)}

Caffey, an eminent American paediatric radiologist, was the first to describe a syndrome occurring mainly in the first six months of life and characterised by tender soft tissue swellings and cortical thickening of the underlying bones. ${ }^{1}$

The first case of Caffey's syndrome to be reported in the United Kingdom was a 2 month old baby boy who was admitted to hospital with a one week history of swelling of the face and fever. ${ }^{2}$ On examination there were moist sounds in the right lung and a tender swelling of the left side of the face. He was given antibacterial treatment without improvement; the fever persisted and after 10 days he was noticed to have generalised lymphadenopathy and a tender swelling over the shaft of the left humerus. Over the next few days similar swellings developed over the right ramus of the mandible, both radii, and both tibiae. Radiographs of the long bones, ribs, pelvis, and mandible showed periosteal elevation and irregular new bone formation. Blood examination showed an anaemia with polymorphonuclear leucocytosis and the blood chemistry was normal apart from a raised alkaline phosphatase. There was no evidence of syphilis, tuberculosis, or scurvy. A biopsy specimen of the left tibia showed proliferation of osteoblasts but no evidence of infection or malignancy. The clinical signs gradually subsided over the next three months, and one year after the onset the child was well and the radiographs showed a return to normal.

\section{Thrush in infancy}

In a 12 month period in Liverpool necropsies were carried out on 204 infants under the age of 1 year, of whom 28 were aged less than 4 weeks. In seven $(25 \%)$ of the neonates and $19(11 \%)$ of the older infants pharyngeal or oesophageal lesions of thrush were found and were considered directly or indirectly responsible for the death of the child. ${ }^{3}$ Of the 26 infants with thrush only three had been breast fed and only two were of low birth weight. The commonest clinical features on admission were oral lesions of thrush, vomiting with or without diarrhoea, and severe dehydration. Otitis media developed later in 10 cases, in four of which Monilia albicans (now Candida albicans), was found on smear or culture.

At postmortem examination 21 of the 26 infants were found to have oesophageal lesions of thrush, some with ulceration or sloughing. Eleven had histological evidence of inhalation of vomit with aspiration pneumonia and six had died suddenly due to obstruction of the airways by aspirated material. In two infants infection had spread from the oesophagus to the pleural cavity causing an empyema. Pyaemic abscesses, which were presumed to have originated in the ulcerated oesophagus, were found in various organs in five cases. In two there were metastatic thrush lesions in the kidneys. Ulcerated areas in the intestine were present in four cases but only in one of these was this proved to be due to thrush.

The authors warn that thrush may pass unnoticed at necropsy unless histological examination is undertaken, as the macroscopical appearances may be non-specific. In their view the histology suggests that monilia is the primary infection and that the damaged epithelium is invaded by bacteria, rather than the monilia being an opportunistic secondary invader of a primary bacterial lesion.

1 Caffey J. Syphilis of the skeleton in early infancy. The nonspecificity of many of the roentgenological changes. $A \mathcal{F} R$ 1939:42:637-55.

2 O'Reilly JN. Infantile cortical hyperostosis. Arch Dis Child 1949;24:67-70.

3 Lederer H, Todd R McL. Thrush in infancy. Arch Dis Child 1949;24:200-7.

A D M JACKSON 